Research Article

\title{
Ableitung der thermischen Bodenfunktionen anhand von Körnung und Porengrößenverteilung als Grundlage für In-situ-Vorhersagen des Bodenwärmehaushaltes
}

\section{Derivation of the thermal soil functions by grain size and pore size distribution as a basis for in situ predictions of the soil heat balance}

\author{
Rainer Horn ${ }^{1 *}$, Yili Lu², Tusheng Ren², Robert Horton ${ }^{3}$, Anneka Mordhorst ${ }^{1}$, Heiner Fleige ${ }^{1}$
}

\author{
${ }^{1}$ Institut für Pflanzenernährung und Bodenkunde, Christian-Albrechts-Universität zu Kiel (CAU Kiel), Hermann-Rodewald-Straße 2, 24118 \\ Kiel, Deutschland \\ ${ }^{2}$ College of Land Science and Technology, China Agricultural University, Beijing, China \\ 3 Department of Agronomy, Iowa State University, Ames, Iowa \\ Korrespondierender Autor: rhorn@soils.uni-kiel.de
}

Einreichung: 1. April 2021, Annahme: 1. Juni 2021

\section{Zusammenfassung}

Zu den Beeinträchtigungen natürlicher Bodenfunktionen auf Leitungsbaustellen (wie z. B. zur Strom- und Fernwärmeversorgung) zählen neben Bodenstruktur- und Porenfunktionsstörungen auch Veränderungen der standortspezifisch variablen thermischhydraulischen Eigenschaften im Bereich der Leitungsgräben.

Auf der Grundlage der in der „KA5“ (Ad-hoc-AG Boden, 2005) zusammengestellten Werte der Porengrößenverteilung für die einzelnen Bodenarten lassen sich für unterschiedliche Trockenrohdichtebereiche mithilfe des mathematischen Modells von Xie et al. (2018) die volumetrische Wärmekapazität $(\mathrm{C})$, Wärme- $(\lambda)$ und Temperaturleitfähigkeit $(\mathrm{k})$ in Abhängigkeit vom Matrixpotenzial (pF-Wert) berechnen. Diese berechneten Daten werden tabellarisch für das Bodenartenspektrum nach KA5 dargestellt sowie exemplarisch für ausgewählte Bodenarten (Sl3, Lt2, Tu3) und Klassenbereiche der Trockenrohdichte veranschaulicht.

Es zeigen sich die typischen Kurvenverläufe für C (linearer Anstieg), $\lambda$ (zunehmend abflachender Anstieg) und $\mathrm{k}$ (Anstieg mit Umkehr zu negativer Steigung) und deren Zunahme mit steigender Trockenrohdichte $\left(1,1-1,7 \mathrm{~g} \mathrm{~cm}^{-3}\right)$. $\mathrm{k}$ variiert unter Berücksichtigung der Anteile aus Ton, Schluff und Sand innerhalb der Bodenarten. Hier unterscheiden sich die Kurvenverläufe zwischen geringer und hoher Trockenrohdichte beispielsweise stärker für „Sl3“ als für die feinkörnigere Bodenart „Tu3“. Mithilfe dieser Datensätze lassen sich u. a. Veränderungen des Wärmehaushaltes und der thermisch-hydraulischen Bodeneigenschaften bei Leitungsvorhaben vor Beginn der Baumaßnahmen prognostizieren, werden insbesondere Bodenart und Trockenrohdichte anhand von Bohrstockkartierungen auf diesen Standorten bestimmt.

Schlagwörter: Bodenarten, Porengrößenverteilung, Modellierung, Wärmeleitfähigkeit, Temperaturleitfähigkeit, Wärmekapazität

\section{Summary}

In addition to soil structure and pore function disturbances, the impairments of natural soil functions on line construction sites (e. g. power cable routing) also include changes in the site-specific variable thermal-hydraulic properties in the area of the line trenches. Based on the pore size distribution values as a function of dry bulk density classes compiled in German Soil Classification system "KA5" (Ad-Hoc-AG-Boden, 2005), volumetric heat capacity (C), thermal conductivity ( $\lambda$ ), and thermal diffusivity (k) can be calculated using the model of Xie et al. (2018). These calculated data are presented for the soil texture spectrum according to KA5, as well for selected textures (Sl3, Lt2, Tu3) and class ranges of dry bulk density $\left(\rho_{\mathrm{t}}\right)$.

The typical curves for $\mathrm{C}$ (linear increase), $\lambda$ (increasingly flattening increase) and $\mathrm{k}$ (increase with reversal to negative slope) and their rise with increasing $\rho \mathrm{t}\left(1.1-1.7 \mathrm{~g} \mathrm{~cm}^{-3}\right)$ are shown. $\mathrm{k}$ varies depending on the proportions of clay, silt and sand within the soil texture class. The curve shapes between low and high $\rho$ tiffer more for "Sl3" than for the finer-grained texture class "Tu3". With the help of these data sets, changes in the heat balance and the thermal- hydraulic soil properties can be predicted for line projects in the power grid expansion before the start of construction measures, if soil texture and dry bulk density are determined by field description methods.

Keywords: Soil texture, pore size distribution, modelling, thermal conductivity, thermal diffusivity, heat capacity

D Open Access. (C) 2021 Rainer Horn, Yili Lu, Tusheng Ren, Robert Horton, Anneka Mordhorst, Heiner Fleige published by Sciendo. (c) Br-Nc-ND This work is licensed under the Creative Commons Attribution-NonCommercial-NoDerivatives 3.0 License.

https://doi.org/10.2478/boku-2021-0004 


\section{Einleitung}

Die Temperaturleitfähigkeit $\mathrm{k}$ des Bodens beschreibt die Geschwindigkeit der Übertragung von Temperaturwellen und bestimmt damit die Tiefe des Bodens, die von der täglichen oder auch saisonalen Oberflächenerwärmung und -abkühlung beeinflusst wird. Sie ist daher je nach volumetrischer Wärmekapazität $(\mathrm{C})$ und Wärmeleitfähigkeit $(\lambda)$ für die Modellierung der Wärmeübertragung aber auch des Wasserflusses in Böden und des Energiehaushalts des Bodens sowie für die Vorhersage der Bodentemperatur an der Landoberfläche auch in agrarisch genutzten Böden unerlässlich. Dies gilt nicht nur im Hinblick auf die durch den Klimawandel zu erwartenden Änderungen der Lufttemperatur, sondern auch auf die gleichzeitigen Auswirkungen auf den Bodenwärmestrom und die entsprechenden gekoppelten Wasser-, Gas- und Nährstoffflüsse - und damit letztendlich auch auf das Pflanzenwachstum sowie die Grundwasserneubildungsrate. $\mathrm{k}$ ist somit zur Abschätzung des Bodenwärmestroms erforderlich, einer wichtigen Komponente der Landoberflächenenergiebilanz, die den Energieaustausch zwischen der Landoberfläche und der Atmosphäre beeinflusst (Heusinkveld et al., 2004).

$\mathrm{Zu}$ diesen längerfristig wirksamen Änderungen kommen weitere Eingriffe in den Boden hinzu, die z. B. durch den Ausbau eines dichten Netzes von Fernwärmeleitungen vor allem in urbanen Räumen (Wessolek et al., 2017) verursacht werden, oder durch die in den nächsten Jahren geplante Verlegung von Stromkabeln zu erwarten sind. Um den in Norddeutschland nachhaltig produzierten Strom bis nach Süddeutschland zu leiten, werden Stromkabel in verschiedenen Trassen quer durch die Bundesrepublik Deutschland (Abbildung1) verlegt. Damit entsteht im Boden eine weitere Abwärme-Energiequelle, durch die zusätzliche hydraulische, aber auch mechanische, biologische und bodengenetische Prozesse induziert werden (Hartge und Horn, 2016). Unter landwirtschaftlichen Aspekten muss dabei, abgesehen von dem enormen mechanischen Eingriff in Bodenstruktur- und ökologische Bodenfunktionen, sowohl während der Erdkabelverlegung als auch nach abgeschlossener Wiederverfüllung der mehrere Meter breiten und ca.1,5-2 m tiefen Erdkabelgräben (vgl. Zink et al., 2013; Horn et al., 2021), zusätzlich die an der Außenseite der Erdkabel auftretende Abwärme von bis $\mathrm{zu}+70^{\circ} \mathrm{C}$ hinsichtlich ihrer Wirkung auf die Prozesse in Böden berücksichtigt werden. Nach den momentan geltenden Eingriffsregelungen in Böden wird die in diesem Zusammenhang relevante Thematik „Bodenschutz“ vornehmlich über das Baugesetzbuch (BauGB) und nur dort, wo das BauGB die Bodenbelange nicht mehr regelt, vom Bundesbodenschutzgesetz (BBodSchG) bzw. von der Bundesbodenschutzverordnung (BBodSchV) abgedeckt.

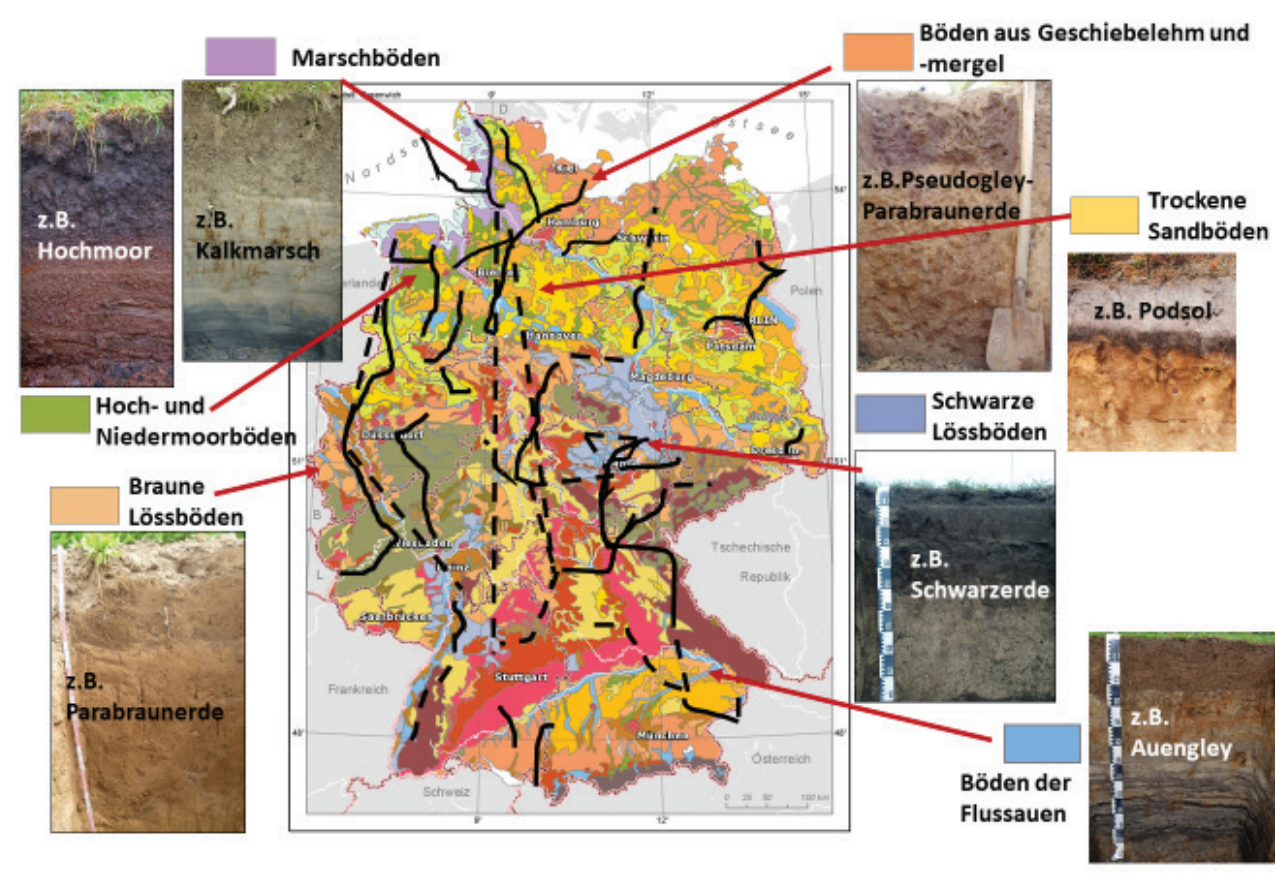

Abbildung 1. Bodenübersichtskarte von Deutschland 1: 5.000.000 (BGR Hannover) einschließlich der Leitungsvorhaben im Stromübertragungsnetz. Figure 1. Soil overview map of Germany 1: 5,000,000 (BGR Hannover) with illustration of line projects in the electricity transmission network. 
Allgemein wird gefordert, dass an der Bodenoberkante möglichst keine Temperaturunterschiede im Vergleich zur unbeeinflussten Bodenfläche auftreten, damit die ökologischen Bodenfunktionen nicht beeinträchtigt werden. Allerdings muss in diesem Zusammenhang auch die Frage beantwortet werden, wie man diese kontinuierlich abstrahlende Wärme vor allem im Untergrund verteilen kann, ohne dass die mit der Landnutzung verbundenen Ansprüche an den Boden, z. B. verstärkte Austrocknung, Abbau der organischen Bodensubstanz, Änderungen der mikrobiologischen Aktivität, Erhöhung der chemischen Reaktionsgeschwindigkeit $\left(\mathrm{Q}_{10}\right.$-Wert) (Winkler et al., 1996; Chen et al. 2020), negativ verändert werden. Die ReaktionsgeschwindigkeitTemperatur-Regel (RGT) besagt, dass sich die Reaktionsgeschwindigkeit etwa verdoppelt $\left(\mathrm{Q}_{10}\right.$-Wert $\left.\approx 2\right)$, wenn die Temperatur um 10 Kelvin (K) bzw. $10{ }^{\circ} \mathrm{C}$ erhöht wird (Temperatur in ${ }^{\circ} \mathrm{C}=$ Temperatur in $\mathrm{K}-273,15$ ).

Durch den mechanischen Eingriff in die Bodenstruktur verändern sich die Standorteigenschaften im Bereich des Erdkabelgrabens im Vergleich zu den natürlichen Böden erheblich. Neben dem veränderten Bodenwasserhaushalt verändert sich auch durch die abstrahlende Wärme der Kabel der Bodenwärmehaushalt (keine oder geringere strukturbildende Frostgare, höhere Evaporation u. a.), sodass Unterschiede im Ertrag zu erwarten sind. Das spiegelt sich u. a. in Luftbildern wider (Gebhardt et al., 2012). Wechselwirkungen zwischen Bodenwasser- und Bodenwärmehaushalt sowie ökosystemare Folgen sollten daher im Vorfeld standortspezifisch quantifiziert werden.

Dies setzt aber die Messung der in situ Wärmehaushaltsparameter und -funktionen voraus, da sowohl $\mathrm{k}$ als auch deren Einzelkomponenten: $\mathrm{C}$ und $\lambda$ eine deutliche Abhängigkeit vom Wassergehalt, der Bodenstruktur und der Porengrößenverteilung je nach Trockenrohdichte aufweisen (Hartge und Horn, 2016). Vor diesem Hintergrund ist es nicht verwunderlich, dass es gerade im Zusammenhang mit den variierenden Standorteigenschaften nur wenige auf In-situ-Bedingungen übertragbare Datensätze gibt (Trinks, 2010; Trinks et al., 2013; Dehner und Feldwisch, 2015; Markert et al., 2016). k kann zwar mittels der stufenweisen Temperaturänderung an der Oberfläche unter Verwendung der analytischen Lösung der eindimensionalen Wärmeleitungsgleichung (für weitere Detailinformationen s. Jackson and Taylor, 1986; Horton, 2002) abgeschätzt werden, allerdings funktioniert sie nach Erfahrungen der Autoren nur an Bodensäulen im Labor, wohingegen sich die Wärmeimpulssonden als zuverlässige Werkzeuge für In-situ-Messungen anbieten (für detailliertere Informationen s. Xie et al., 2018). Allerdings sind die dafür notwendigen Messpunkte bei den über mehrere $1000 \mathrm{~km}$ langen Leitungen schwer zu realisieren.

Xie et al. (2018) haben auf der Grundlage einer intensiven Literaturrecherche eine indirekte Methode zur Berechnung von $\mathrm{k}$ mithilfe der Bodentextur und Porengrößenverteilung entwickelt und in Form eines Modells an insgesamt 8 natürlich strukturierten Bodenproben getestet. Hierbei haben sie C nach de Vries (1963) sowie $\lambda$ nach dem Verfahren von Lu et al. (2007) berücksichtigt.

Ziel dieser Arbeit ist es mit dem Verfahren von Xie et al. (2018) die thermischen Kenngrößen, $C, \lambda$ und $\mathrm{k}$ für das Bodenartenspektrum (gemäß deutscher Klassifikation nach Ad-hoc-AG-Boden, 2005) und der davon abhängigen Porengrößenverteilungen bei unterschiedlicher Trockenrohdichte zu berechnen, um somit eine Grundlage für eine zukünftig standortspezifische Bodenwärmehaushaltsanalytik zu schaffen.

\section{Material und Methoden}

Es ist bekannt, dass die Temperaturleitfähigkeit $\mathrm{k}$ als Quotient aus Wärmeleitfähigkeit $\lambda$ und volumetrischer Wärmekapazität $\mathrm{C}$ mit der Bodentextur, dem Wassergehalt $(\Theta)$ und der Trockenrohdichte $\left(\rho_{t}\right)$ variiert. Jedoch gibt es nur wenige Modelle, die diese Beziehung genau quantifizieren. In dieser Publikation wurde das empirische Modell von Xie et al. (2018) zur Abschätzung der Temperaturleitfähigkeit und der einzelnen thermischen Bodenfunktionen aus der Partikelgrößenverteilung des Bodens, der $\rho_{\mathrm{t}}$ und dem Grad der Wassersättigung $S_{r}$ anhand der Porengrößenverteilung herangezogen (Gleichung 1).

$$
k\left(S_{r}\right)=\frac{0,25+\exp \left(b-S_{r}^{-a}\right)}{4,18 S_{r}+c} \quad S_{r}>0 \quad \text { Gleichung } 1
$$

Die Modellparameter a, b und c sind abgeleitete Kurvenparameter der $\mathrm{k}\left(\mathrm{S}_{\mathrm{r}}\right)$-Funktion aus Xie et al. (2018), die über die Anpassung der Gleichungen an Wärmeimpuls/Temperaturleitfähigkeits-Daten für 8 strukturierte Böden bestimmt und einen weiten Bereich von Körnung, $\rho_{\mathrm{t}}$ und Wassersättigungsgrad abdecken. Der Wert 4,18 (MJ m$\left.{ }^{-3} \mathrm{~K}^{-1}\right)$ beschreibt die Wärmekapazität von Wasser bei Raumtemperatur (Campbell et al., 1991) und $0,25\left(\mathrm{~W} \mathrm{~m}^{-1} \mathrm{~K}^{-1}\right)$ ist der Wert für die mittlere Wärmeleitfähigkeit trockener mineralischer Böden $\left(\lambda_{\text {dry }}\right)$ (de Vries, 1963). $S_{\mathrm{r}}$ wurde aus dem Verhältnis zwischen $\Theta$ und der Porosität berechnet unter Annahme der Festsubstanzdichte von Quarz $\left(2,65 \mathrm{~g} \mathrm{~cm}^{-3}\right)$. 
Xie et al. (2018) konnten nachweisen, dass das neue Modell die Temperaturleitfähigkeit/Sättigungsgrad-Beziehung genau beschreibt und damit nützliche Schätzungen aus dem Sättigungsgrad, der $\rho_{\mathrm{t}}$ und der Bodentextur liefert. Als Berechnungsgrundlagen wurden in der vorliegenden Arbeit ebenfalls die in Xie et al. (2018) kombinierten Ansätze von de Vries (1963) zur Berechnung der volumetrischen Wärmekapazität (Gleichung 2) und von Lu et al. (2007) (Gleichung 3) zur Bestimmung der Wärmeleitfähigkeit verwendet.

$$
\begin{aligned}
& C=\rho_{t} c_{s}+\rho_{w} c_{w} \theta \\
& \lambda=\exp \left(\beta-\theta^{-\alpha}\right)+\lambda_{d r y} \quad \theta>0 \quad \text { Gleichung } 3
\end{aligned}
$$

Die Variablen $\mathrm{c}_{\mathrm{s}}$ und $\mathrm{c}_{\mathrm{w}}$ geben die spezifische Wärme der Festphase bzw. von Wasser $\left(4,18 \mathrm{~g}^{-1} \mathrm{~K}^{-1}\right)$ an. Die Dichte von Wasser $\left(\rho_{\mathrm{w}}\right)$ wird mit $1,0 \mathrm{~g} \mathrm{~cm}^{-3}$ berechnet. $\alpha$ und $\beta$ sind Kurvenparameter des empirischen Modells von $\mathrm{Lu}$ et al. (2014) in Abhängigkeit von der Porengrößenverteilung und $\rho_{t}$. Die Wärmeleitfähigkeit des trockenen Bodens $\left(\lambda_{\text {dry }}\right)$ lässt sich aus der Porosität berechnen (siehe oben).

Für die in dieser Arbeit durchgeführten Berechnungen von $\lambda$, C und $\mathrm{k}$ wurden die in der „KA5“ (Ad-Hoc-AG Boden 2005) zusammengestellten Bodenarten (KA5-Tab. 30) in Abhängigkeit von der Porengrößenverteilung und der Trockenrohdichte (KA5-Tab. 70) als Eingangskenngrößen verwendet Die volumetrischen Wassergehalte bei $\mathrm{pF}$ 2,5 in „KA5“ wurden auf der Grundlage der KA5-Tab. 75 für die einzelnen Trockenrohdichteklassen als Schätzwerte eingesetzt.

Die Bodenarten wurden nach KA5-Tab. 30 weiter differenziert, um auch die Spannbreiten in der Korngrößenzusammensetzung innerhalb einer Texturklasse (Bodenart) abzubilden. Es wurde unterteilt in eine:

- mittlere Korngrößenzusammensetzung (mittlere Gehalte an Sand, Schluff und Ton)

- Korngrößenzusammensetzung mit maximalen Tongehalten und variierenden Schluffgehalten

- Korngrößenzusammensetzung mit minimalen Tongehalten und variierenden Schluffgehalten.

Für die weiterführende Berechnung gehen die typischen Kennwerte: Gesamtporenvolumen (entspricht der Wassersättigung $=\mathrm{pF}-\infty)$ sowie die weiteren volumetrischen Wassergehalte bei den Matrixpotenzialen (pF-Werten): $\mathrm{pF}$ 1,8 (d. h. die weiten Grobporen sind entwässert), pF 2,5 (d. h. die weiten und engen Grobporen sind entwässert) und $\mathrm{pF}$
4,2 (entspricht den mit nicht pflanzenverfügbarem Bodenwasser gefüllten Feinporen) von einzelnen Bodenarten differenziert in die 3 Trockenrohdichteklassen: $\rho_{\mathrm{t}}<1,4 \mathrm{~g} \mathrm{~cm}^{-3}$, $1,4-1,6 \mathrm{~g} \mathrm{~cm}^{-3}$ sowie $>1,6 \mathrm{~g} \mathrm{~cm}^{-3}$ ein. Dabei wurden die trockenrohdichteabhängigen Porengrößenverteilungen und daraus berechneten Sättigungsgrade nach der mittleren Korngrößenverteilung herangezogen, da in der „KA5“ keine weitere Differenzierung innerhalb der Texturklassen (minimale und maximale Tongehalte) vorgenommen wurde.

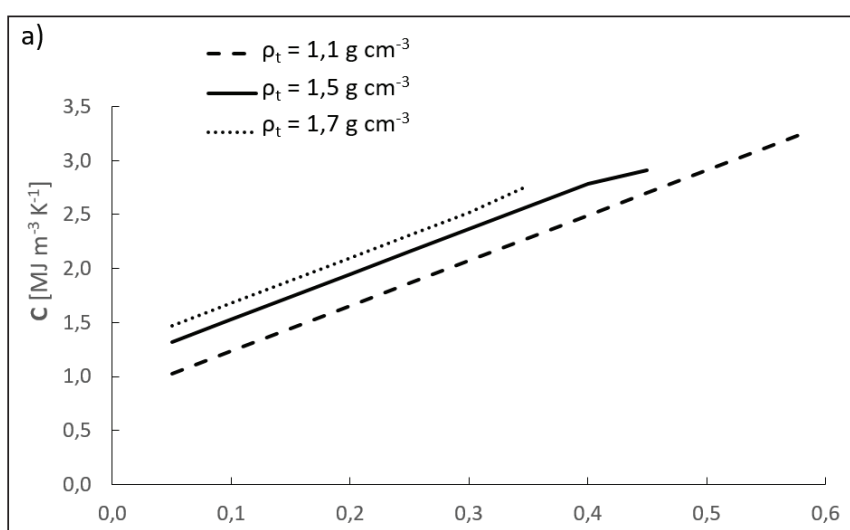

b)
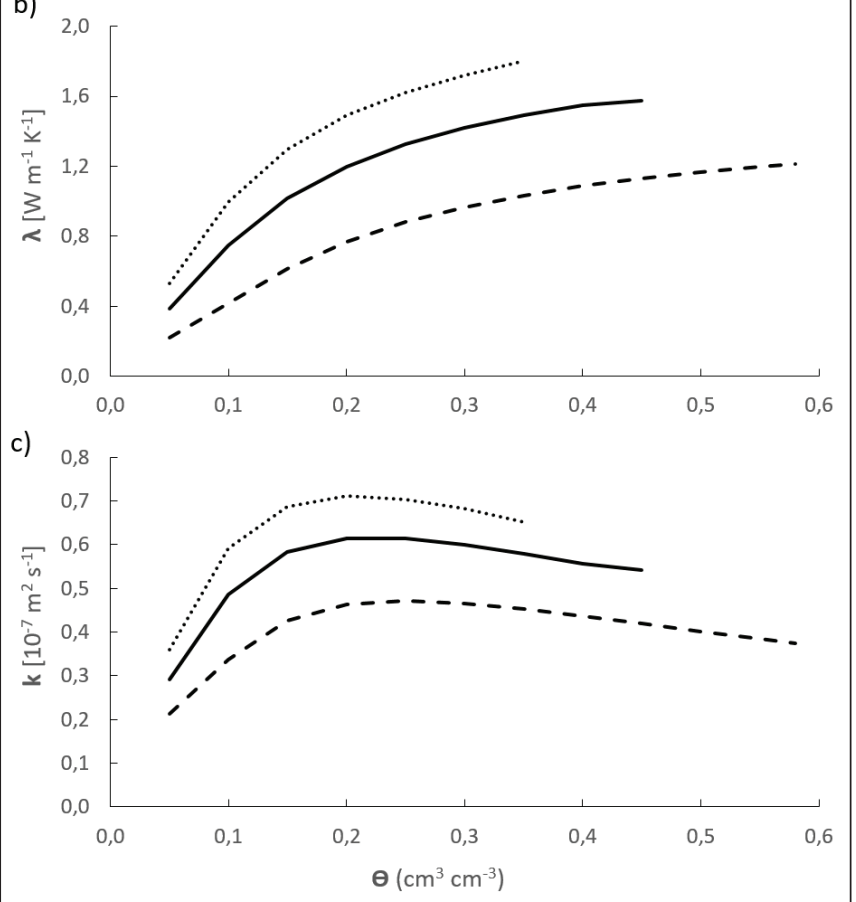

Abbildung 2. Änderung der volumetrischen Wärmekapazität C (a), der Wärmeleitfähigkeit $\lambda$ (b) und der Temperaturleitfähigkeit k (c) mit dem volumetrischen Wassergehalt $\Theta$ und der Trockenrohdichte $\rho$ t für die Bodenart Lt2.

Figure 2. Changes in volumetric heat capacity $\mathrm{C}$ (a), thermal conductivity $\lambda$ (b) and thermal diffusivity $\mathrm{k}$ (c) with volumetric water content $\Theta$ and bulk density $\rho$ t for the German Soil texture class Lt2. 


\section{Ergebnisse}

In den folgenden Tabellen werden die für jede Bodenart und für verschiedene Bereiche der Trockenrohdichte modellierten Kennwerte: volumetrische Wärmekapazität $(C)$, Wärmeleitfähigkeit $(\lambda)$ und Temperaturleitfähigkeit (k) bei unterschiedlichem Matrixpotenzial: $\mathrm{pF}-\infty, \mathrm{pF}$ 1,8, pF 2,5 und pF 4,2 aufgelistet. Die modellierten Kennwerte für C (Tabelle 1), $\lambda$ (Tabelle 2) und $\mathrm{k}$ (Tabelle 3) werden zudem bodenartspezifisch bei der jeweils mittleren Korngrößenzusammensetzung (Tabelle 1-3 a), bei maximalen (Tabelle 1-3 b) und minimalen Tongehalten der jeweiligen Bodenart (Tabelle 1-3 c) dargestellt.

In Abbildung 2a-c werden exemplarisch für die Bodenart „schwach toniger Lehm“ (Lt2) die Beziehungen zwischen $\Theta, \rho_{\mathrm{t}}$ und den thermischen Bodenfunktionen $\mathrm{C}, \lambda$ und $\mathrm{k}$ veranschaulicht. In Abbildung $2 \mathrm{a}$ ist der linearer Anstieg der $\mathrm{C}$ mit steigendem $\Theta$ zu erkennen, während Abbildung $2 b$ zeigt, wie der Verlauf der $\lambda$ nach anfänglich steilerem Anstieg bei höheren $\Theta$-Werten abflacht. k stellt den typischen Kurvenverlauf mit anfänglich steilem Anstieg und leichtem Absinken bei höherem $\Theta$ (negative Steigung) dar (Abbildung 2c). Mit zunehmender $\rho_{\mathrm{t}}$ steigen bei vergleichbarem $\Theta$ (bzw. Wassersättigung) die Werte der 3 thermischen Bodenkenngrößen $\lambda, \mathrm{C}$ und $\mathrm{k}$ an.

\section{Bodenart: Tu3}
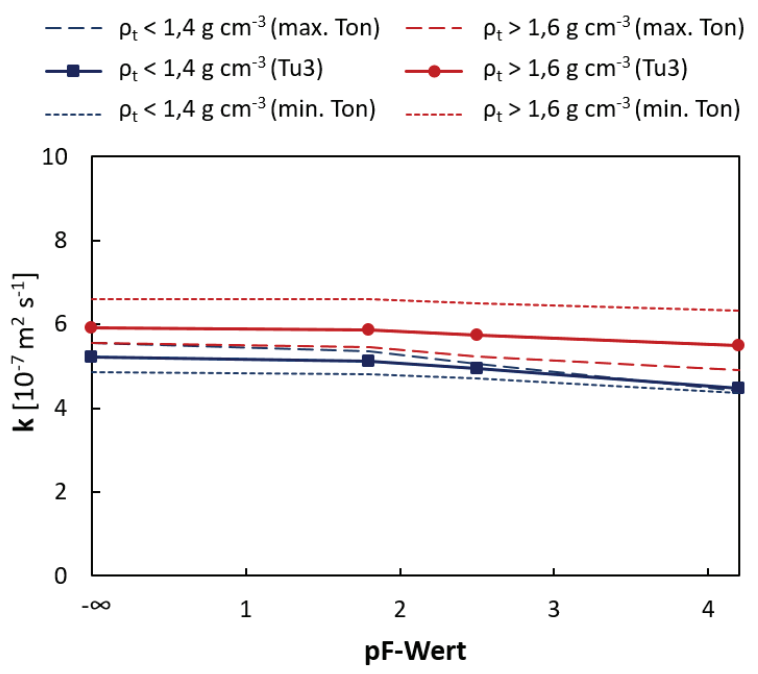

Abbildung 3. Einfluss des Tongehaltes bei der Bodenart Tu3 auf die Unterschiede in der Temperaturleitfähigkeit $\mathrm{k}$ bei unterschiedlichen Trockenrohdichten und Matrixpotenzialen (pF-Wert).

Figure 3. Impact of clay content across the German soil texture class Tu3 on differences in the thermal diffusivity $\mathrm{k}$ depending on bulk density and matric potential ( $\mathrm{pF}$-value).
Als Beispiele für die deutlich variierenden thermischen Kennwerte ist der Einfluss des Tongehaltes innerhalb der Bodenart „stark schluffiger Ton“ Tu3 (Abbildung 3) und „stark lehmiger Sand“ Sl3 (Abbildung 4) auf den Verlauf von $\mathrm{k}$ für 2 unterschiedliche Trockenrohdichtebereiche $\left(\rho_{\mathrm{t}}<1,4 \mathrm{~g} \mathrm{~cm}^{-3}\right.$ und $\left.>1,6 \mathrm{~g} \mathrm{~cm}^{-3}\right)$ mit zunehmendem Matrixpotenzial (pF-Wert) dargestellt. Generell ist zu erkennen, dass $\mathrm{k}$ bei höherer $\rho_{\mathrm{t}}$ größer wird als bei geringer $\rho_{\mathrm{t}}$ und dass innerhalb der Bodenart Sl3 bei hohen Tongehalten

Tabelle 1a. Einfluss der Bodenarten (BA bei mittlerer Korngrößenzusammensetzung) auf die volumetrische Wärmekapazität $\mathrm{C}$ in Abhängigkeit vom Matrixpotenzial (pF-Wert) für die 3 Klassen der Trockenrohdichte $<1,4 \mathrm{~g} \mathrm{~cm}^{-3}, 1,4-1,6 \mathrm{~g} \mathrm{~cm}^{-3}$ und $>1,6 \mathrm{~g} \mathrm{~cm}^{-3}$

Table 1a. Impact of soil texture classes (BA with mean particle size distribution) on the volumetric heat capacity $\mathrm{C}$ depending on matric potential ( $\mathrm{pF}$ value) for the three bulk density classes $<1.4 \mathrm{~g} \mathrm{~cm}^{-3}, 1.4-1.6 \mathrm{~g} \mathrm{~cm}^{-3}$ und $>1.6 \mathrm{~g} \mathrm{~cm}^{-3}$

\begin{tabular}{|c|c|c|c|c|c|c|c|c|c|c|c|c|}
\hline & \multicolumn{4}{|c|}{$\begin{array}{c}\mathrm{C}\left[\mathrm{MJ} \mathrm{m} \mathrm{m}^{-3} \mathrm{~K}^{-1}\right] \\
\text { bei } \rho_{\mathrm{t}}<1,4 \mathrm{~g} \mathrm{~cm}^{-3}\end{array}$} & \multicolumn{4}{|c|}{$\begin{array}{c}\mathrm{C}\left[\mathrm{MJ} \mathrm{m} \mathrm{m}^{-3} \mathrm{~K}^{-1}\right] \\
\text { bei } \rho_{\mathrm{t}}=1,4-1,6 \mathrm{~g} \mathrm{~cm}^{-3}\end{array}$} & \multicolumn{4}{|c|}{$\begin{array}{c}\mathrm{C}\left[\mathrm{MJ} \mathrm{m}^{-3} \mathrm{~K}^{-1}\right] \\
\text { bei } \rho_{\mathrm{t}}>1,6 \mathrm{~g} \mathrm{~cm}^{-3}\end{array}$} \\
\hline BA & $\mathrm{pF}$ & $\mathrm{pF}$ & $\mathrm{pF}$ & $\mathrm{pF}$ & $\mathrm{pF}$ & $\mathrm{pF}$ & $\mathrm{pF}$ & $\mathrm{pF}$ & $\mathrm{pF}$ & pF & $\mathrm{pF}$ & $\mathrm{pF}$ \\
\hline BA & $-\infty$ & 1,8 & 2,5 & 4,2 & $-\infty$ & 1,8 & 2,5 & 4,2 & $-\infty$ & 1,8 & 2,5 & 4,2 \\
\hline Ss & 3,07 & 1,57 & 1,19 & 1,19 & 2,92 & 1,50 & 1,41 & 1,29 & 2,79 & 1,66 & 1,49 & 1,36 \\
\hline Su2 & 3,07 & 2,07 & 1,61 & 1,23 & 2,94 & 2,06 & 1,52 & 1,27 & 2,76 & 2,14 & 1,76 & 1,43 \\
\hline $\mathrm{Sl} 2$ & 3,10 & 2,13 & 1,63 & 1,30 & 2,92 & 2,17 & 1,83 & 1,41 & 2,76 & 2,22 & 1,84 & 1,51 \\
\hline $\mathrm{Sl} 3$ & 3,12 & 2,37 & 1,70 & 1,45 & 2,92 & 2,29 & 1,83 & 1,50 &, 74 & 2,32 & 1,95 & 1,61 \\
\hline St2 & 3,07 & 2,49 & 1,65 & 1,32 & 2,90 & 2,06 & 1,73 & 1,39 &, 70 & 2,07 & 1,78 & 1,53 \\
\hline Su3 & 3,12 & 2,41 & 1,82 & 1,36 & 2,92 & 2,33 & 1,96 & 1,46 & 2,76 & 2,35 & 2,01 & 1,51 \\
\hline Su4 & 3,14 & 2,55 & & 1, & 2,92 & 2,46 & 2,08 & 1, & 76 & 2,43 & 14 & \\
\hline Slu & 3,12 & 2,53 & 2,11 & 1,57 & 2,92 & 2,50 & 2,21 & 1,62 & 2,79 & 2,49 & 2,24 & 1,70 \\
\hline S14 & 3,16 & 2, & 1, & 1,4 & 2,90 & 2,39 & 2,10 & 1,6 & 2,72 & 2,38 & 2,22 & 1,76 \\
\hline St3 & 3,14 & & 2,09 & $1,($ & 2,94 & 2,36 & 2,10 & 1,7 & 74 & 2,36 & 2,16 & 1,86 \\
\hline Ls2 & 3,14 & 2,60 & 2,09 & 1,7 & 2,92 & 2,54 & 2,17 & 1,8 & 2,79 & 2,53 & 2,16 & 1,95 \\
\hline Ls3 & 3,16 & 2, & 1,99 & $1,($ & 2,90 & 2,52 & 2,14 & 1,8 & .76 & 2, & 2,14 & 1,93 \\
\hline Ls4 & 3,16 & 53 & 2,07 & 1,7 & 2,92 & 2,46 & 2,08 & 1,7 & 2,74 & 2,45 & 2,20 & 1,91 \\
\hline Lt2 & 3,14 & 2,68 & 2,26 & 1,93 & 2,92 & 2,63 & 2,42 & 2,04 & 2,79 & 2,58 & 2,41 & 2,12 \\
\hline Lts & 3,19 & & & & 2,96 & 2,71 & 2,46 & 2,1 & 81 & 2,60 & 2,43 & \\
\hline Ts4 & 3,21 & 2,66 & 2,24 & 1,9 & 2,90 & 2,48 & 2,39 & 1,89 & 2,76 & 2,51 & 2,30 & 2,05 \\
\hline Ts3 & 3,15 & 2,8 & 2,44 & 2,1 & 2,96 & 2,71 & 2,58 & 2,16 & 83 & 2,62 & 2,41 & 2,16 \\
\hline $\mathrm{Uu}$ & 3,14 & & 2 & 1,4 & 2,96 & 2,67 & 2,42 & & 81 & 2,68 & 2,22 & \\
\hline Us & 3,12 & 2,66 & 2,20 & 1,49 & 2,94 & 2,56 & 2,31 & 1,52 & 2,76 & 2,60 & 2,39 & 1,68 \\
\hline Ut2 & 3,07 & 2,66 & 2,11 & 1,4 & 2,92 & 2,67 & 2,42 & 1, & 81 & 2,68 & 2,31 & 1,72 \\
\hline Ut3 & 3,07 & 2,61 & 2,20 & 1,5 & 2,92 & 2,67 & 2,46 & 1,62 & 2,81 & 2,68 & 2,26 & 1,7 \\
\hline Uls & 3,12 & 2,57 & 2,16 & 1,57 & 2,92 & 2,58 & 2,37 & 1,66 & 2,81 & 2,60 & 2,22 & 1,72 \\
\hline Ut4 & 3,10 & 2,59 & 2,26 & 1,63 & 2,94 & 2,65 & 2,48 & & 2,81 & 2,68 & 2,35 & \\
\hline $\mathrm{Lu}$ & 3,14 & 2,64 & 2,18 & 1,76 & 2,92 & 2,63 & 2,33 & 1,91 & 2,79 & 2,62 & 2,20 & 1,99 \\
\hline Lt3 & 3,17 & 2,84 & 2,46 & 2,1 & 2,98 & 2,77 & 2,56 & 2,27 & 2,85 & 2,72 & 2,52 & 2,31 \\
\hline Tu3 & 3,17 & 2,84 & 2,54 & 2,13 & 2,98 & 2,73 & 2,60 & 2,18 & 2,85 & 2,72 & 2,52 & 2,31 \\
\hline Tu4 & 3,10 & 2,68 & 2,38 & 1,88 & 2,92 & 2,67 & 2,50 & 1,96 & 2,81 & 2,68 & 2,39 & 2,01 \\
\hline Ts2 & 3,15 & 2,94 & 2,48 & 2,27 & 2,96 & 2,79 & 2,71 & 2,25 & 2,83 & 2,70 & 2,41 & 2,20 \\
\hline $\mathrm{Tl}$ & 3,17 & 2,96 & 2,54 & 2,34 & 3,00 & 2,83 & 2,67 & 2,29 & 2,85 & 2,72 & 2,52 & 2,27 \\
\hline Tu2 & 3,15 & 2,94 & 2,57 & 2,27 & 3,02 & 2,85 & 2,73 & 2,35 & 2,87 & 2,75 & 2,54 & 2,33 \\
\hline $\mathrm{Tt}$ & 3,21 & 3,05 & 2,67 & 2,42 & 3,02 & 2,90 & 2,73 & 2,35 & 2,83 & 2,75 & 2,49 & 2,24 \\
\hline
\end{tabular}


ein Anstieg von k zu höheren Matrixpotenzialen (pF-Werten) verschoben ist. Außerdem ist zu erkennen, dass die Kurvenverläufe zwischen geringer und höherer $\rho_{\mathrm{t}}$ deutlich unterschiedlicher für die Bodenart Sl3 als Tu3 ausfallen. So betragen die größten Unterschiede zwischen geringer und hoher $\rho_{\mathrm{t}}$ bei der Bodenart Sl3 etwa 3 log-Einheiten von $\mathrm{k}$, bei der Bodenart Tu3 nur die Hälfte (1,5 log-Einheit k bei minimalem Tongehalt innerhalb der Bodenart).

Tabelle 1b. Einfluss der Bodenarten (BA bei maximalem Tongehalt) auf die volumetrische Wärmekapazität $\mathrm{C}$ in Abhängigkeit vom Matrixpotenzial (pF-Wert) für die 3 Klassen der Trockenrohdichte $<1,4 \mathrm{~g} \mathrm{~cm}^{-3}$, $1,4-1,6 \mathrm{~g} \mathrm{~cm}^{-3}$ und $>1,6 \mathrm{~g} \mathrm{~cm}^{-3}$

Table 1b. Impact of soil texture classes (BA with maximal clay content) on the volumetric heat capacity $\mathrm{C}$ depending on matric potential ( $\mathrm{pF}$ value) for the three bulk density classes $<1,4 \mathrm{~g} \mathrm{~cm}^{-3}, 1,4-1,6 \mathrm{~g} \mathrm{~cm}^{-3}$ und $>1,6 \mathrm{~g} \mathrm{~cm}^{-3}$

\begin{tabular}{|c|c|c|c|c|c|c|c|c|c|c|c|c|}
\hline & \multicolumn{4}{|c|}{$\begin{array}{c}\mathrm{C}\left[\mathrm{MJ} \mathrm{m} \mathrm{m}^{-3} \mathrm{~K}^{-1}\right] \\
\text { bei } \rho_{\mathrm{t}}<1,4 \mathrm{~g} \mathrm{~cm}^{-3}\end{array}$} & \multicolumn{4}{|c|}{$\begin{array}{c}\mathrm{C}\left[\mathrm{MJ} \mathrm{m}^{-3} \mathrm{~K}^{-1}\right] \\
\text { bei } \rho_{\mathrm{t}}=1,4-1,6 \mathrm{~g} \mathrm{~cm}^{-3}\end{array}$} & \multicolumn{4}{|c|}{$\begin{array}{c}\mathrm{C}\left[\mathrm{MJ} \mathrm{m} \mathrm{m}^{-3} \mathrm{~K}^{-1}\right] \\
\text { bei } \rho_{\mathrm{t}}>1,6 \mathrm{~g} \mathrm{~cm}^{-3}\end{array}$} \\
\hline & $\mathrm{pF}$ & E & 5 & & $\mathrm{pF}$ & $\mathrm{pF}$ & 5 & $\mathrm{nF}$ & $F$ & 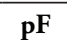 & $\mathrm{nF}$ & $\mathrm{nF}$ \\
\hline BA & $-\infty$ & 1,8 & & & $-\infty$ & & & & $-\infty$ & & & \\
\hline Ss & 3,07 & 1,57 & 1,19 & 1,19 & 2,92 & 1,50 & 1,41 & 1,29 & 2,79 & 1,66 & 1,49 & \\
\hline Su2 & & & & & & & & & & & & \\
\hline $\mathrm{Sl} 2$ & 10 & & & & 2,92 & & & & & & & \\
\hline $\mathrm{Sl} 3$ & 3,1 & & & & 2,92 & & & & & & & \\
\hline St2 & & & & & ,90 & & & & & & & \\
\hline $\mathrm{Su}$ & & & & & ,92 & & & & & & & \\
\hline Su4 & & & & & 2,92 & & & & 76 & & & \\
\hline Slu & & & & & 92 & & & & & & & \\
\hline S14 & 3,16 & & & & 2,90 & & & & & & & \\
\hline St3 & 4 & & & & 2,94 & & & & 74 & & & \\
\hline Ls & & & & & 92 & & & & & & & \\
\hline Ls3 & 3,1 & & & & 2,90 & & & & 2,76 & & & \\
\hline Ls4 & & & & & & & & & & & & \\
\hline Lt2 & & & & & 2,96 & & & & & & & \\
\hline Lts & 3,19 & 2,7 & & & 2,96 & 2 , & & & 81 & 2,60 & 2,43 & \\
\hline Ts 4 & & & & & & & & & & & & \\
\hline Ts3 & 3,15 & & & & 2,96 & & & & 33 & & & \\
\hline $\mathrm{Uu}$ & , 1 & & & & 2,96 & & & & 81 & 2,6 & & \\
\hline Us & 3,12 & & & & 2,94 & & & & & & & \\
\hline Ut2 & 3,07 & 2,66 & 2,11 & & 2,92 & 2,67 & 2,42 & & 2,81 & 2,68 & 2,31 & \\
\hline $\mathrm{Ut}$ & & & & & 2,92 & & & & & & & \\
\hline Uls & 3,12 & 2,57 & 2,16 & 1,57 & 2,92 & 2,58 & 2,37 & 1,66 & 2,81 & 2,60 & 2,22 & \\
\hline Ut4 & 3,10 & & 2,2 & & 2,94 & 2,65 & 2 , & & 2,81 & 2,68 & 2,35 & \\
\hline $\mathrm{Lu}$ & & & & & 2,92 & 2,63 & & & & & & \\
\hline Lt3 & 3,17 & 2,84 & 2,46 & & 2,98 & 2,77 & 56 & 27 & 2,85 & 2,72 & 2,52 & \\
\hline Tu3 & & & & & 2,98 & 2,73 & & & 35 & 2,72 & 2,52 & \\
\hline Tu 4 & 3,13 & 2,71 & 2,42 & & 2,96 & 2,71 & 2,54 & 2,00 & 2,85 & 2,72 & 2,43 & \\
\hline Ts2 & 3,15 & 2,94 & 2,48 & & 2,96 & 2,79 & 2,71 & & 2,83 & 2,70 & 2,41 & \\
\hline $\mathrm{Tl}$ & 3,17 & 2,96 & 2,54 & & 3,00 & 2,83 & 2,67 & & 2,85 & 2,72 & 2,52 & \\
\hline Tu2 & 3,15 & 2,94 & 2,57 & 2,2 & 3,02 & 2,85 & 2,73 & 2,35 & 2,87 & 2,75 & 2,54 & \\
\hline $\mathrm{Tt}$ & 3,21 & 3,05 & 2,67 & 2,42 & 3,02 & 2,90 & 2,73 & 2,35 & 2,83 & 2,75 & 2,49 & 2,24 \\
\hline
\end{tabular}

\section{Diskussion}

Es ist allgemein anerkannt, dass Böden je nach Trockenrohdichte und Bodenstruktur in Abhängigkeit von der Körnung unterschiedliche Fließ- und Speichereigenschaften aufweisen und dabei zusätzlich auch die Anordnung der Partikel und die Porenkontinuität im Boden eine entscheidende Rolle spielen. Betrachtet man in diesem Zusammenhang die Wärmehaushaltskenngrößen:

Tabelle 1c. Einfluss der Bodenarten (BA bei minimalem Tongehalt) auf die volumetrische Wärmekapazität $\mathrm{C}$ in Abhängigkeit vom Matrixpotenzial (pF-Wert) für die 3 Klassen der Trockenrohdichte $<1,4 \mathrm{~g} \mathrm{~cm}^{-3}$, $1,4-1,6 \mathrm{~g} \mathrm{~cm}^{-3}$ und $>1,6 \mathrm{~g} \mathrm{~cm}^{-3}$

Table 1c. Impact of soil texture classes (BA with minimum clay content) on the volumetric heat capacity $\mathrm{C}$ depending on matric potential ( $\mathrm{pF}$ value) for the three bulk density classes $<1.4 \mathrm{~g} \mathrm{~cm}^{-3}, 1.4-1.6 \mathrm{~g} \mathrm{~cm}^{-3}$ und $>1.6 \mathrm{~g} \mathrm{~cm}^{-3}$

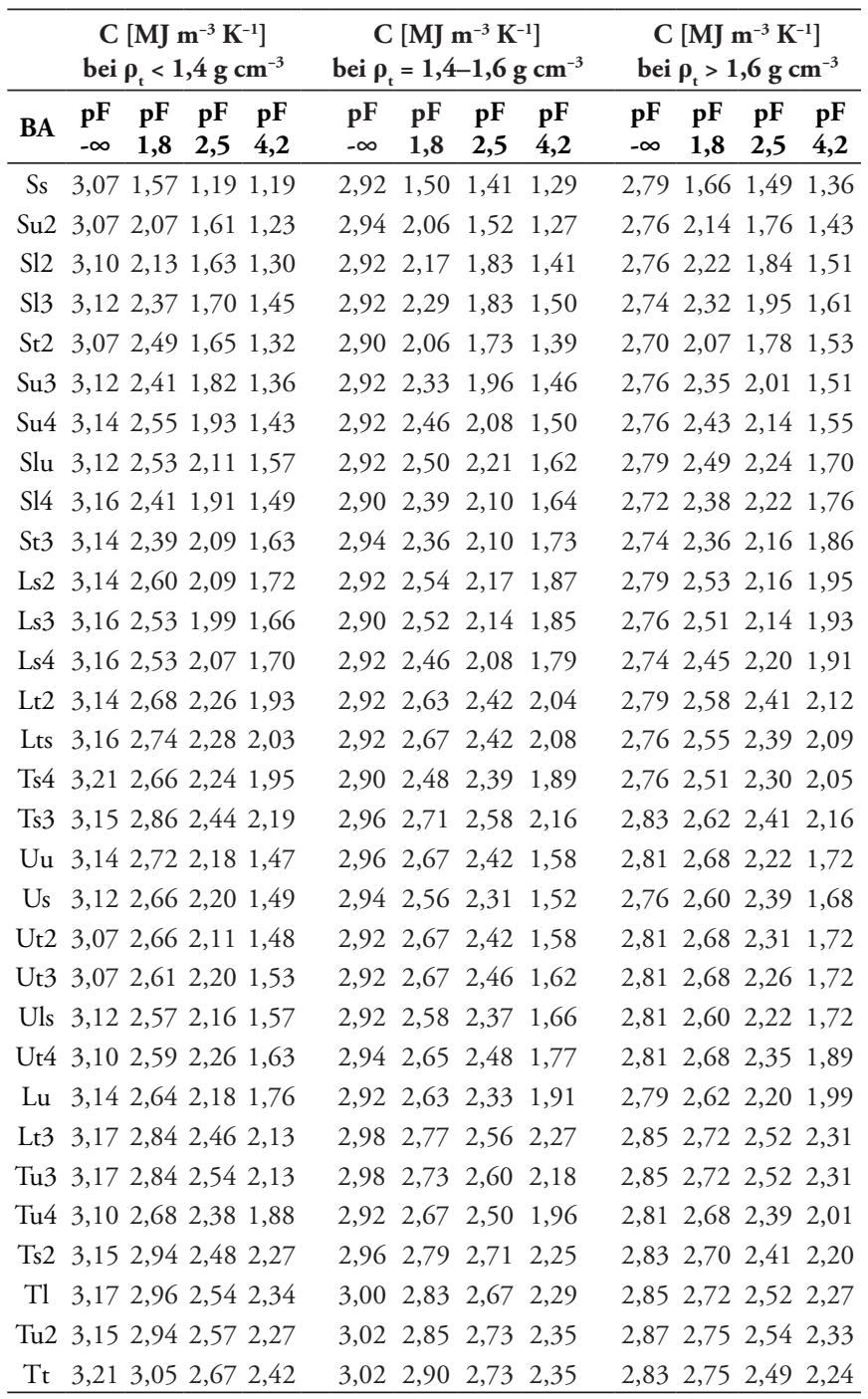


volumetrische Wärmekapazität, Wärme- und Temperaturleitfähigkeit, so lassen sich mittels der Gleichungen von de Vries (1963) für die volumetrische Wärmekapazität, von Xie et al. (2007) für die Wärmeleitfähigkeit sowie von Xie et al. (2018) für die Temperaturleitfähigkeit plausible Kurvenverläufe für verschiedene Körnungen und Trockenrohdichten in Abhängigkeit vom Wassergehalt oder Matrixpotenzial bzw. dem Sättigungsgrad bestimmen.

Die exemplarisch berechneten Kurven für die verschiedenen Trockenrohdichte-Klassen (aus der „KA5“) zeigen

Tabelle 2a. Einfluss der Bodenarten (BA bei mittlerer Korngrößenzusammensetzung) auf die Wärmeleitfähigkeit $\lambda$ in Abhängigkeit vom Matrixpotenzial (pF-Wert) für die 3 Klassen der Trockenrohdichte $<1,4 \mathrm{~g} \mathrm{~cm}^{-3}, 1,4-1,6 \mathrm{~g} \mathrm{~cm}^{-3}$ und $>1,6 \mathrm{~g} \mathrm{~cm}^{-3}$

Table 2a. Impact of soil texture classes (BA with mean particle size distribution) on the thermal conductivity $\lambda$ depending on matric potential ( $\mathrm{pF}$ value) for the three bulk density classes $<1.4 \mathrm{~g} \mathrm{~cm}^{-3}, 1.4-1.6 \mathrm{~g} \mathrm{~cm}^{-3}$ und $>1.6 \mathrm{~g} \mathrm{~cm}^{-3}$

\begin{tabular}{|c|c|c|c|c|c|c|c|c|c|c|c|c|}
\hline & & [W] & & & $\begin{array}{r}\lambda \\
\text { bei } \rho^{\lambda}\end{array}$ & [W & & $\begin{array}{l}-1] \\
\mathrm{g} \mathrm{cm}^{-3}\end{array}$ & & [ & 6 & \\
\hline $\mathbf{D A}$ & $\mathrm{pF}$ & $\mathrm{pF}$ & & $\mathrm{pF}$ & $\mathrm{pF}$ & $\mathrm{pF}$ & & $\mathrm{pF}$ & $\mathrm{pF}$ & $\mathrm{pF}$ & & $\mathrm{pF}$ \\
\hline & & & & & & & & & & & 2,5 & \\
\hline Ss & 2,03 & 1,24 & 0,73 & 0,73 & 2,42 & 1,28 & ,13 & & 2,80 & 1,68 & 1,30 & 0,8 \\
\hline Su2 & 1,85 & & & 0,75 & & 1,70 & 19 & 0,74 & & & 1,69 & \\
\hline $\mathrm{Sl} 2$ & 1,74 & & & 0,81 & 07 & 1,73 & 49 & 0,99 & 42 & & 1,72 & 1,1 \\
\hline $\mathrm{Sl} 3$ & & & & & & 1,69 & & & & & 1,76 & \\
\hline St2 & 1,91 & & & & & 1,84 & & & & & ,89 & 1,28 \\
\hline Su3 & & & & & & & & & & & 1,77 & \\
\hline Su4 & & & & & & & & & & & & \\
\hline Slu & 1,44 & & & & 1,70 & 1,56 & 44 & 1,07 & 90 & 78 & 1,65 & \\
\hline S14 & & & & & & 1,72 & & & & & & \\
\hline St3 & 1,65 & & & & & 1,77 & 64 & & & & & \\
\hline Ls2 & 1,30 & & & & & 1,44 & 1 , & 1,1 & 70 & 1,62 & 1,45 & \\
\hline Ls3 & & & & & & 1, & & & & 77 & ,57 & \\
\hline Ls4 & 1,44 & & & & & 1,62 & 44 & & & & & \\
\hline Lt2 & 1,27 & & & 0 , & 1,49 & 1,42 & 1,36 & 1,1 & 64 & 1,58 & 1,52 & 1 \\
\hline Lts & 1,3 & & & & & & & & & & & \\
\hline Ts4 & 1,46 & 1,3 & & 1,1 & 1,94 & 1,78 & 1,75 & 1,4 & & 2,08 & 1, & \\
\hline Ts3 & 1,49 & & & & & 1,70 & 65 & 1,4 & & 91 & 81 & \\
\hline $\mathrm{Uu}$ & 1,30 & & & & & 1,43 & 1 , & & & & & \\
\hline Us & 1,34 & 1,25 & & 0,6 & 1,53 & 1,44 & 1,36 & 0,72 & 76 &, 71 & 1,63 & 0 \\
\hline Ut2 & 1,43 & & & & & & & & & & & \\
\hline Ut3 & 1,39 & 1,30 & 1,17 & 0,72 & 1,57 & 1,51 & 1,45 & 0,87 & ,71 & 1,68 & 1,52 & 1, \\
\hline Uls & 1,30 & 1,20 & & 0,7 & & 1,42 & 1,36 & 0,8 & ,63 & 1,57 & 1,42 & \\
\hline Ut4 & 1,34 & 1,24 & & & & 1,44 & 1,39 & 1,00 & & 1, & & \\
\hline $\mathrm{Lu}$ & 1,38 & 1,29 & 1,15 & 0,93 & 1,66 & 1,58 & 1,48 & 1,23 & 85 & 1,80 & 1,60 & \\
\hline Lt3 & 1,38 & & & 1,1 & & 1,58 & 1,52 & 1,40 & & 1,7 & 1,71 & \\
\hline Tu3 & 1,30 & 1,25 & 1,18 & 1,05 & 1,51 & 1,46 & 1,42 & 1,27 & 1,67 & 1,64 & $: 1,57$ & \\
\hline Tu4 & 1,32 & 1,24 & 1, & 0, & 1,50 & 1,44 & 1,40 & 1,15 & 63 & 1,59 & 1,50 & \\
\hline Ts2 & 1,36 & 1,32 & & 1,15 & & 1,55 & 1,52 & 1,36 & 1,76 & 1,72 & 1,61 & \\
\hline $\mathrm{Tl}$ & 1,21 & 1,18 & 1,10 & 1,0 & 1,37 & 1,34 & 1,30 & 1,19 & 1,52 & 1,49 & 1,43 & 1,3 \\
\hline Tu2 & 1,31 & 1,2 & 1,2 & & & 1,41 & 1,38 & 1,27 & 1,62 & 1,59 & 1,52 & 1,4 \\
\hline $\mathrm{Tt}$ & 1,23 & 1,20 & 1,14 & 1,08 & 1,42 & 1,40 & 1,36 & 1,25 & 1,64 & 1,62 & 1,54 & 1,45 \\
\hline
\end{tabular}

ähnliche Verläufe wie die von Wessolek et al. (2017) und decken sich auch mit den in verschiedenen Lehrbüchern (z. B. Scheffer/Schachtschabel, 2018, Hartge und Horn, 2016) beschriebenen Zusammenhängen. Geht man daher von den in der „KA5“ auf der Grundlage mehrerer Tausend Datensätze zur Porengrößenverteilung sowie der als repräsentativ definierten mittleren Körnung der insgesamt jeweils 31 Bodenarten aus und kombiniert diese beiden Datensätze als Eingangsgrößen für die Modellierung der Wärmehaushaltskenngrößen, dann erhält man mit den bekannten Modellansätzen Zahlenwerte, die für die

Tabelle 2b. Einfluss der Bodenarten (BA bei maximalem Tongehalt) auf die Wärmeleitfähigkeit $\lambda$ in Abhängigkeit vom Matrixpotenzial (pF-Wert) für die 3 Klassen der Trockenrohdichte $<1,4 \mathrm{~g} \mathrm{~cm}-3,1,4-1,6 \mathrm{~g} \mathrm{~cm}^{-3}$ und $>1,6 \mathrm{~g} \mathrm{~cm}^{-3}$

Table $2 \mathrm{~b}$. Impact of soil texture classes (BA with maximum clay content) on the thermal conductivity $\lambda$ depending on matric potential ( $\mathrm{pF}$ value) for the three bulk density classes $<1.4 \mathrm{~g} \mathrm{~cm}^{-3}, 1.4-1.6 \mathrm{~g} \mathrm{~cm}^{-3}$ und $>1.6 \mathrm{~g} \mathrm{~cm}^{-3}$

\begin{tabular}{|c|c|c|c|c|c|c|c|c|c|c|c|c|}
\hline & \multicolumn{4}{|c|}{ 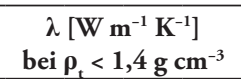 } & \multicolumn{4}{|c|}{ 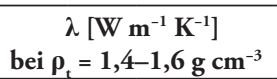 } & \multicolumn{4}{|c|}{$\begin{array}{c}\lambda\left[\mathrm{W} \mathrm{m}^{-1} \mathrm{~K}^{-1}\right] \\
\text { bei } \rho_{\mathrm{t}}>1,6 \mathrm{~g} \mathrm{~cm}^{-3}\end{array}$} \\
\hline & $\mathrm{pF}$ & $\mathrm{pF}$ & $\mathrm{pF}$ & & $\mathrm{pF}$ & $\mathrm{pF}$ & $\mathrm{pF}$ & $\mathrm{pF}$ & pF & & $\mathrm{pF}$ & \\
\hline BA & & & & & & & & & & & 2,5 & \\
\hline Ss & 2,05 & 1,25 & 0,73 & 0,73 & 44 & 1,29 & 13 & 0,8 & 2,83 & 1,69 & 1,31 & \\
\hline $\mathrm{Su} 2$ & 1,74 & 1,39 & & & & & 12 & & & & 57 & \\
\hline $\mathrm{Sl} 2$ & 1,67 & & & & 98 & 5 & 42 & & 30 & & & \\
\hline $\mathrm{Sl} 3$ & & & & & & & & & & & & \\
\hline St2 & 1,78 & 60 & & & & & 41 & & & & & \\
\hline Su3 & 48 & & & & & & 36 & & 2 & & 1,60 & \\
\hline Su4 & & & & & & & & & & & & \\
\hline Slu & 1,31 & 20 & & 0,74 & 1,5 & 1,42 & & 0 , & 58 & 1 & 48 & \\
\hline S14 & & & & & & & & & & & & \\
\hline St3 & & & & & & & & & & & & \\
\hline Ls2 & ,23 & & & & & 5 & 11 & 1, & 57 & & 1,35 & \\
\hline Ls3 & 29 & & & & & & & & & 1 & & \\
\hline Ls4 & 37 & & & & & & & & 4 & & & \\
\hline Lt2 & 1,35 & 1,27 & & & 1 , & & 47 & & 80 & 73 & 66 & \\
\hline Lts & 1,21 & & & & & & & & & & & \\
\hline Ts4 & 1,46 & 1,34 & & & 1,9 & & & 12 & 20 & 2 & & \\
\hline Ts3 & 37 & & & & & & & & & 1 & & \\
\hline $\mathrm{Uu}$ & 1,27 & 1,20 & & & & & & 0 , & & & & \\
\hline Us & 1,36 & 1,27 & & 0, & 1,56 & 1,47 & 1,38 & 0,7 & 80 & 1,74 & 1,66 & \\
\hline Ut2 & & & & & & & & & & & & \\
\hline Ut3 & 1,36 & 1,27 & 1,15 & 0,71 & 1,53 & 1,47 & 1,41 & 0,85 & ,67 & 1,63 & 1,47 & \\
\hline Uls & 1,40 & & & & & & 1, & 0 & 80 & 1 & 36 & \\
\hline Ut4 & ,34 & & & & & & & & & & & \\
\hline $\mathrm{Lu}$ & 1,30 & 1,21 & 1, & 0,8 & 1,53 & 1,46 & 1,37 & 1,14 & ,69 & 1,65 & 1,47 & \\
\hline $\mathrm{Lt} 3$ & 1,30 & & & & & 1,46 & & & & 1 , & 1,56 & \\
\hline Tu3 & 1,27 & 1,21 & 1,15 & 10 & 1,47 & 1,41 & 1,38 & 1,23 & 1,62 & 1,5 & 1,52 & \\
\hline Tu4 & 1,34 & 1,26 & 19 & 0,9 & & 1,47 & 1,43 & 1,17 & 1,67 & 1,63 & 1,53 & \\
\hline Ts2 & 1,41 & 1,37 & & & & 1,62 & 1,60 & & 1,85 & 1,81 & 1,69 & \\
\hline $\mathrm{Tl}$ & 1,30 & 1,26 & & 1, & 1,48 & 1,45 & 1,41 & 1,28 & 1,67 & 1,63 & 1,56 & \\
\hline Tu2 & 1,29 & 1,26 & & 1,1 & 1,4 & 1,39 & 1,36 & 1,25 & 1,59 & 1,56 & 1,50 & 1,4 \\
\hline $\mathrm{Tt}$ & 1,23 & 1,20 & 1,14 & 1,08 & 1,42 & 1,40 & 1,36 & 1,25 & 1,64 & 1,62 & 1,54 & 1,43 \\
\hline
\end{tabular}


Modellierung von gekoppelten thermischen und hydraulischen Prozessen in unterschiedlich texturierten Böden herangezogen werden können.

Im Hinblick auf die eingangs erwähnte Stromkabeltrassenproblematik gewährleistet dieser Ansatz somit eine gute Grundlage, um die durch die Wärmeabstrahlung von Stromkabeln in Böden unter verschiedenen hydraulischen Bedingungen im Jahresverlauf zu erwartenden zusätzlichen Bodenaustrocknungen zu prognostizieren. Außerdem lassen sich mithilfe dieser Datensätze Austrocknungszustände im Trassenbereich, die z. B. eine zeitlich frühere „Not"reife der Bestände bedingen, prognostizieren bzw. quantifizieren. Auch weitere Maßnahmen für die Folgebewirtschaftung von derartigen Trassenverläufen können auf diese Weise definiert werden. Es ist außerdem denkbar, dass mit den bekannten gekoppelten thermisch-hydraulischen Bodenwasserhaushaltsmodellen entsprechende Ertragsprognosen für den Landwirt z. B. hinsichtlich Wassermangels erarbeitet werden können, wobei die theoretischen Grundlagen auch bei Ehlers (1996) sowie Kirkham (2004) zusammenfassend dargestellt werden.

Allerdings kann bei der gegenwärtigen Datenlage der für die Ertragsentwicklung sowie Wasser- und Nährstoffspeicherung wesentliche Bodenstruktureinfluss bei den vorliegenden Berechnungen nicht berücksichtigt werden,

\section{Bodenart: Sl3}

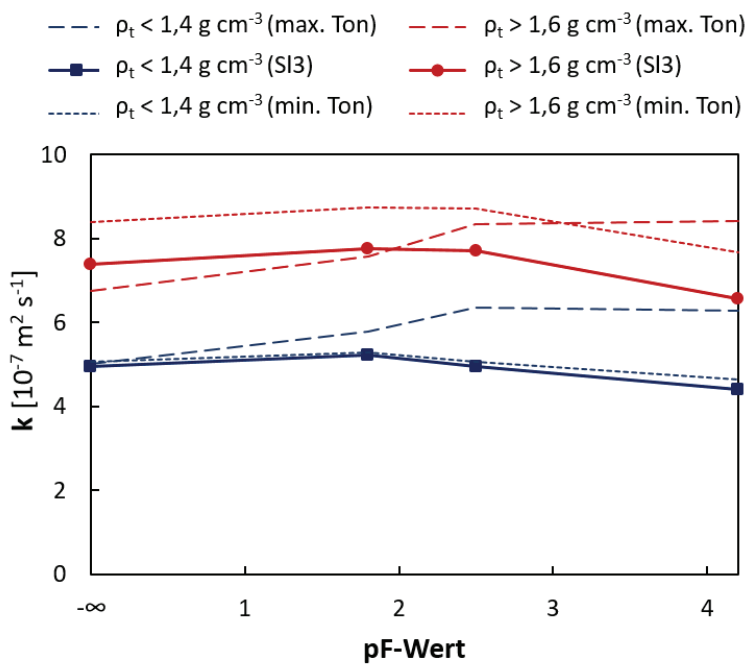

Abbildung 4. Einfluss des Tongehaltes in der Bodenart Sl3 auf die Unterschiede in der Temperaturleitfähigkeit bei unterschiedlichen Trockenrohdichten und Matrixpotenzialen (pF-Wert).

Figure 4. Impact of clay content across the German soil texture class Tu3 on differences in the thermal diffusivity $\mathrm{k}$ depending on bulk density and matric potential ( $\mathrm{pF}$-value). da dieser lediglich überschlägig anhand der von der Trockenrohdichte abhängigen Porengrößenverteilung abgeleitet wird. Kaune et al. (1993) konnten den Einfluss der Bodenstruktur für die Wärmeausbreitung und Folgeerscheinungen anhand der Versuche unter ariden Klimabedingungen in der Negev Wüste (Israel) dokumentieren. Es ist davon auszugehen, dass bei einer stabilen Bodenstruktur z. B. Subpolyeder oder Krümelstruktur die Kontinuität der Bodenporen ebenso wie die Anordnung der Bodenpartikel im System weitgehend optimiert sind. Folglich muss mit einer noch gleichmäßigeren Wärmeausbreitung

Tabelle 2c. Einfluss der Bodenarten (BA bei minimalem Tongehalt) auf die Wärmeleitfähigkeit $\lambda$ in Abhängigkeit vom Matrixpotenzial (pF-Wert) für die 3 Klassen der Trockenrohdichte $<1,4 \mathrm{~g} \mathrm{~cm}^{-3}, 1,4-1,6 \mathrm{~g} \mathrm{~cm}^{-3}$ und $>1,6 \mathrm{~g} \mathrm{~cm}^{-3}$

Table 2c. Impact of soil texture classes (BA with minimum clay content) on the thermal conductivity $\lambda$ depending on matric potential ( $\mathrm{pF}$ value) for the three bulk density classes $<1.4 \mathrm{~g} \mathrm{~cm}^{-3}, 1.4-1.6 \mathrm{~g} \mathrm{~cm}^{-3}$ und $>1.6 \mathrm{~g} \mathrm{~cm}^{-3}$

\begin{tabular}{|c|c|c|c|c|c|c|c|c|c|c|c|c|}
\hline & & $\begin{array}{l}\text { [W } \\
\rho_{\epsilon}\end{array}$ & $\begin{array}{l}\mathrm{n}^{-1} \mathrm{I} \\
1,4 \mathrm{~g}\end{array}$ & $\mathrm{~m}^{-3}$ & bei $\rho$ & $\begin{array}{l}\text { [W } \\
=1\end{array}$ & 1 & $\mathbf{m}^{-3}$ & & [V & & \\
\hline & $\mathrm{pF}$ & $\mathrm{pF}$ & $\mathrm{pF}$ & $\mathrm{pF}$ & $\mathrm{pF}$ & $\mathrm{pF}$ & $\mathrm{pF}$ & & $\mathrm{pF}$ & & & $\mathrm{pF}$ \\
\hline & & & & & & & & & & 1,8 & 2,5 & \\
\hline Ss & 1,99 & 1,22 & 0,71 & 0,71 & 2,36 & 1,26 & 1,10 & & 2,73 & 1,64 & 1,27 & \\
\hline Su2 & 1,99 & & 1,26 & 0 & 31 & 1,84 & 1,28 & & 80 & & & \\
\hline $\mathrm{Sl} 2$ & 1,88 & & 1 , & 0,87 & 2,27 & 1,89 & 1,62 & 1,07 & 2,68 & 2,31 & 1,90 & 1,2 \\
\hline $\mathrm{Sl} 3$ & ,80 & & & & ,22 & 1, & 59 & & 58 & & & \\
\hline St2 & 2,05 & 1 & 1,33 & & ,50 & 1,99 & 1,65 & & 12 & & & \\
\hline Su3 & 72 & & & & 2,11 & & 51 & & & & & \\
\hline Su4 & & & & & 88 & & & & & & & \\
\hline Slu & 1,48 & & & 0, & ,76 & 1,6 & 1, & & & & & \\
\hline S14 & 1,68 & & & & ,20 & & & & & & & \\
\hline St3 & 1,76 & & 1,41 & 1,1 & ,17 & 1,91 & 1,76 & $1,4 C$ & 2,68 & 2,43 & 2,25 & \\
\hline Ls2 & 47 & & & & ,78 & 1,65 & 1,49 & & 00 & & 1 & \\
\hline Ls3 & , 44 & & & & ,81 & 1,68 & & & & & & \\
\hline Ls4 & 1,58 & 1,4 & 1,26 & 1,0 & 2,00 & 1,81 & 1,61 & & 2,37 & 2 , & 2,03 & \\
\hline Lt2 & 1,39 & 0 & & 1, & 67 & & 51 & & 87 & 79 & 1 & \\
\hline Lts & & & 1,28 & & & & 1,69 & & & & & \\
\hline Ts4 & 55 & 1,4 & 1,29 & 1 , & 2,10 & 1,93 & 1,88 & & 2,40 & 2,26 & 2,13 & \\
\hline Ts3 & & & & & & & & & & & & \\
\hline $\mathrm{Uu}$ & ,38 & & & 0,6 & 1,60 & 1,53 & 1,45 & & & & & \\
\hline Us & 1,45 & & & 0,8 & 68 & 1 , & 47 & & 95 & 1,88 & 1 & \\
\hline Ut2 & & & 1,07 & & 1,46 & 1, & & & & & & \\
\hline Ut3 & 1,27 & 1,19 & 1,07 & 0,67 & 1,41 & 1,36 & 1,30 & 0,80 & 1,52 & 1,49 & 1,35 & 4 \\
\hline Uls & 39 & 1,27 & & 0,8 & 1,63 & 1,5 & 1,46 & & 79 & 1,7 & & \\
\hline Ut4 & 1,42 & 1,32 & 1,22 & 0,8 & 1,61 & 1,54 & 1,49 & & 1,80 & 1,76 & 1,63 & \\
\hline $\mathrm{Lu}$ & 1,29 & 1,20 & 1,07 & 0,8 & 1,52 & 1,46 & 1,36 & 1,13 & 1,68 & 1,64 & 1,46 & \\
\hline Lt3 & 1,31 & & & & & 1,48 & 1,42 & & 1,69 & & & \\
\hline Tu3 & 1,35 & 1,30 & 1,23 & 1,09 & 1,59 & 1,53 & 1,49 & 1,32 & 1,77 & 1,73 & 1,66 & \\
\hline Tu4 & 37 & 1,29 & 1,22 & 1,0 & 7 & 1,5 & 1,46 & 1,2 & 1,71 & 1,6 & 1,58 & \\
\hline Ts2 & 1,49 & 1,45 & 1,33 & 1,26 & 1,78 & 1,73 & 1,70 & 1,51 & 2,00 & 1,95 & 1,81 & \\
\hline $\mathrm{Tl}$ & 1,35 & & 1,23 & 1,1 & 1,55 & 1,52 & 1,48 & 1,3 & 1,76 & 1,72 & 1,65 & \\
\hline Tu2 & 1,25 & 1,22 & 1,14 & 1,06 & 1,37 & 1,34 & 1,31 & 1,21 & 1,53 & 1,50 & 1,44 & 1,36 \\
\hline $\mathrm{Tt}$ & 1,23 & 1,20 & 1,14 & 1,08 & 1,42 & 1,40 & 1,36 & 1,25 & 1,64 & 1,62 & & \\
\hline
\end{tabular}


gerechnet werden als wenn bei gleicher Trockenrohdichte die vorhandenen Poren tortuos und diskontinuierlich im Boden vorliegen (Horn und Smucker, 2005). Dies ist bei der Interpretation der Daten für die Prognose der Temperaturausdehnung in gestörtem oder z. B. wiederverfülltem Boden hinsichtlich der Übertragbarkeit auf die angrenzenden, nicht beeinflussten Böden zu berücksichtigten. Vor dem Hintergrund dieser Ergebnisse kann der von Xie et al. (2018) entwickelte Modellansatz, der auf in situ durchgeführten Messungen an strukturierten Böden unter

Tabelle 3a. Einfluss der Bodenarten (BA bei mittlerer Korngrößenzusammensetzung) auf die Temperaturleitfähigkeit k in Abhängigkeit vom Matrixpotenzial (pF-Wert) für die 3 Klassen der Trockenrohdichte $<1,4 \mathrm{~g} \mathrm{~cm}^{-3}$, $1,4-1,6 \mathrm{~g} \mathrm{~cm}^{-3}$ und $>1,6 \mathrm{~g} \mathrm{~cm}^{-3}$

Table 3a. Impact of soil texture classes (BA with mean particle size distribution) on the thermal diffusivity $\mathrm{k}$ depending on matric potential ( $\mathrm{pF}$ value) for the three bulk density classes $<1.4 \mathrm{~g} \mathrm{~cm}^{-3}, 1.4-1.6 \mathrm{~g} \mathrm{~cm}^{-3}$ und $>1.6 \mathrm{~g} \mathrm{~cm}^{-3}$

\begin{tabular}{|c|c|c|c|c|c|c|c|c|c|c|c|c|}
\hline & \multicolumn{4}{|c|}{$\begin{array}{c}\mathbf{k}\left[10^{-7} \mathrm{~m}^{2} \mathrm{~s}^{-1}\right] \\
\text { bei } \rho_{\mathrm{t}}<1,4 \mathrm{~g} \mathrm{~cm}^{-3}\end{array}$} & \multicolumn{4}{|c|}{$\begin{array}{c}\mathrm{k}\left[10^{-7} \mathrm{~m}^{2} \mathrm{~s}^{-1}\right] \\
\text { bei } \rho_{\mathrm{t}}=1,4-1,6 \mathrm{~g} \mathrm{~cm}^{-3}\end{array}$} & \multicolumn{4}{|c|}{$\begin{array}{c}\mathrm{k}\left[10^{-7} \mathrm{~m}^{2} \mathrm{~s}^{-1}\right] \\
\text { bei } \rho_{\mathrm{t}}>1,6 \mathrm{~g} \mathrm{~cm}^{-3}\end{array}$} \\
\hline & pF & $\mathbf{p F}$ & & & pF & $\mathbf{p F}$ & $\mathbf{p F}$ & pF & & $\mathrm{pF}$ & pF & $\mathrm{pF}$ \\
\hline BA & $-\infty$ & & & & & & & & & & & \\
\hline Ss & 5,58 & & 77 & 6,77 & 08 & 8,82 & 8,80 & 8,53 & 8,67 & & & 10 , \\
\hline Su2 & & & & & & & & & & & & \\
\hline $\mathrm{Sl} 2$ & & & & & & & 6,96 & & & & & \\
\hline $\mathrm{Sl} 3$ & & & & & & & & & & & & \\
\hline St2 & & & & & & 6, & & & & & & \\
\hline Su3 & & & & & & & & & & & & \\
\hline Su4 & & & & & & & & & & & & \\
\hline Slu & 5,03 & & & & & 6,46 & 6,92 & & & 7, & & \\
\hline S14 & & & & & & & & & & & & \\
\hline St3 & & & & & & & & & & & & \\
\hline Ls2 & 40 & & & & & & & & & & & 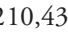 \\
\hline Ls3 & & & & & & & & & & & & \\
\hline Ls4 & & & & & & & & & & & & \\
\hline Lt2 & 4,45 & & & & & & & & & 79 & 89 & \\
\hline & & & & & & & & & & & & \\
\hline Ts4 & 4,5 & & & & & & & & & & & \\
\hline Ts3 & & & & & & & & & & 6,7 & & \\
\hline $\mathrm{Uu}$ & 5,23 & & & & & & & & & & & \\
\hline Us & 4,61 & & & & & 7,68 & 8,36 & 11,17 & 10,18 & & & 18 \\
\hline & & & & & & & & & & & & \\
\hline Ut3 & 5,26 & & & & & 5,88 & & & 6,20 & 6,26 & 6,35 & \\
\hline Uls & 4,63 & & & & & & & & 41 & 8,79 & 9,4 & \\
\hline Ut4 & 35 & & & & & & & & & & & \\
\hline $\mathrm{Lu}$ & 4,68 & 4,78 & 4,73 & 4,3 & & 6,27 & 29 & & 7,27 & 7,36 & 7,40 & 7,19 \\
\hline & 4,6 & & & & & & & & & 7,0 & & \\
\hline Tu3 & 5,23 & & & & & & 5,46 & & 5,92 & 5,87 & 5,73 & \\
\hline lu &, 50 & & & & & & & & 5,60 & 5,58 & 5,4 & \\
\hline Ts2 & 4,62 & & 4,55 & & & & 7,42 & & 10,11 & 101 & 6100 & \\
\hline $\mathrm{Tl}$ & 4,60 & & & 4, & & 5,8 & 5,72 & & & 7,31 & 7,07 & 6,53 \\
\hline Tu2 & 5,40 & & & 4,3 & & 5,4 & 5,27 & 4, & 70 & 5,58 & 5,30 & 4,88 \\
\hline $\mathrm{Tt}$ & 5,55 & אנ, & 4,67 & 4,08 & 5,55 & 5,35 & 5,04 & 4,06 & 5,55 & 5,39 & 4,77 & 3,87 \\
\hline
\end{tabular}

verschiedenen landwirtschaftlichen Bearbeitungen ebenso wie auf Messungen an gestört eingefüllten Böden unter Laborbedingungen beruht, für die modellmäßige Berechnung der thermischen Kenngrößen verwendet werden. Betrachtet man allerdings die Bedeutung der Tongehalte in Kombination mit der Trockenrohdichte innerhalb der jeweiligen Bodenarten für die Temperaturleitfähigkeit, so zeigen sich weitere Interaktionen. So werden die Unterschiede zwischen den Kurvenverläufen bei minimalem und maximalem Tongehalt und beim Vergleich zwischen geringer und hoher Trockenrohdichte umso geringer, je

Tabelle 3b. Einfluss der Bodenarten (BA bei maximalem Tongehalt) auf die Temperaturleitfähigkeit $\mathrm{k}$ in Abhängigkeit in Abhängigkeit vom Matrixpotenzial (pF-Wert) für die 3 Klassen der Trockenrohdichte $<1,4 \mathrm{~g} \mathrm{~cm}^{-3}$, $1,4-1,6 \mathrm{~g} \mathrm{~cm}^{-3}$ und $>1,6 \mathrm{~g} \mathrm{~cm}^{-3}$

Table $3 \mathrm{~b}$. Impact of soil texture classes (BA with maximal clay content) on the thermal diffusivity $\mathrm{k}$ depending on matric potential ( $\mathrm{pF}$ value) for the three bulk density classes $<1.4 \mathrm{~g} \mathrm{~cm}^{-3}, 1.4-1.6 \mathrm{~g} \mathrm{~cm}^{-3}$ und $>1.6 \mathrm{~g} \mathrm{~cm}^{-3}$

\begin{tabular}{|c|c|c|c|c|c|c|c|c|c|c|c|c|}
\hline & $\begin{array}{r}k \\
\text { bei } \rho\end{array}$ & $\begin{array}{l}{\left[10^{-7}\right.} \\
\rho_{t}<1\end{array}$ & & & bei $\rho_{t}$ & {$[10$} & $-1,0 \mathrm{~g}$ & & & $\rho_{t}>1$ & $\mathrm{~s}^{-1}$ & $\begin{array}{l}-1 \\
\mathrm{~cm}^{-3}\end{array}$ \\
\hline & $\mathrm{pF}$ & $\mathrm{pF}$ & $\mathrm{pF}$ & $\mathrm{pF}$ & $\mathrm{pF}$ & $\mathrm{pF}$ & $\mathrm{pF}$ & $\mathrm{pF}$ & $\mathrm{pF}$ & $\mathrm{pF}$ & $\mathrm{pF}$ & $\mathrm{pF}$ \\
\hline & $-\infty$ & 1,8 & 2,5 & 4,2 & & 1,8 & & & & 1,8 & 2,5 & \\
\hline Ss & 5,60 & 6,00 & 5,00 & 5,00 & 7,12 & 7,37 & 7,07 & 6,2 & 8,75 & 9,33 & 8,67 & 7,36 \\
\hline Su2 & 5,22 & & & 5,75 & 6,08 & 7,08 & 7,28 & 6,3 & 7,45 & 8,53 & 9,00 & 8, \\
\hline $\mathrm{Sl} 2$ & 5,07 & & & 4, & & & 6,7 & & 7,30 & 7,92 & 7,99 & \\
\hline $\mathrm{Sl} 3$ & 5,01 & & & 6,27 & 5,86 & 6,76 & 7,38 & 7,24 & 6,74 & 7,58 & 8,35 & 8,41 \\
\hline St2 & 5,2 & & & & 6,49 & & 6,29 & 4, &, 24 & 8,56 & 7,98 & \\
\hline Su3 & 4,97 & & & & & & & 6 , & 73 & 7,46 & 8,02 & \\
\hline Su4 & 5,03 & & & 7,13 & 5,86 & 6,65 & 7,42 & 8,27 &, 51 & 7,27 & 8,05 & 9 , \\
\hline Slu & 4,62 & & & 5,15 & 6,94 & 7,46 & 7,79 & 7,69 & 9,12 & 9,68 & 10 & 10 \\
\hline S14 & 4,9 & & & & & & 18 & & & 44 & & \\
\hline St3 & 4,83 & & & 4,53 & 5,88 & 6 , & 6,10 & 5,6 & 7,15 & 7,43 & 7,46 & 7,10 \\
\hline Ls2 & 4,55 & & & 4,28 & 6,41 & 6 , & 6,58 & 6,2 & 88 & 8,06 & 8,10 & 7,8 \\
\hline Ls3 & 4,19 & & & 4,42 & 7,46 & 7,89 & 8,19 & 8,09 & 9 , & 10,42 & & \\
\hline Ls4 & 4,89 & & & 5 & 5,85 & 6,34 & 6,64 & 6,6 & 6,67 & 7,11 & 7,44 & 7, \\
\hline Lt2 & 4,8 & & & & & & 79 & & 74 & 71 & 6,61 & \\
\hline Lts & 4,39 & & & 3,79 & 6,41 & 6,37 & 6,21 & 5,6 & 16 & 8,10 & 7,96 & 7, \\
\hline Ts4 & 4,51 & & & 4,17 & 6,18 & 6,23 & 6,21 & 5,55 & 08 & 7,14 & 7,09 & 6 , \\
\hline Ts3 & 4,64 & & & 4,7 & 7,60 & & 7,84 & 7,7 & 10,58 & 82 & 10,94 & \\
\hline $\mathrm{Uu}$ & 5,55 & 5,6 & & 4,76 & 5,55 & 5,62 & 5,64 & 4,88 &, 55 & 5,59 & 5,62 & 5,1 \\
\hline Us & 5,05 & & & 4,6 & 5,76 & & 6,01 & & 57 & 69 & 6,81 & \\
\hline Ut2 & 5,19 & & & 4,77 & 5,89 & 6,02 & 6,12 & 5,4 & 6,44 & 6,52 & 6,71 & 6,3 \\
\hline Ut3 & 5,39 & & & 4,40 & 5,66 & 5,70 & 5,71 & 4,73 & 85 & 5,89 & 5,87 & 5,10 \\
\hline Uls & 4,86 & & & & 6,0 & 6,2 & & 5,7 & & 7,02 & 7,14 & \\
\hline Ut4 & 5,35 & 5,30 & 5,13 & 4,14 & 5,62 & 5,60 & 5,55 & 4,63 & 5,85 & 5,85 & 5,75 & 5,09 \\
\hline $\mathrm{Lu}$ & 5,28 & & & 4,1 & 5,66 & 5,5 & 5,41 & 4,7 & 89 & 5,86 & 5,54 & \\
\hline Lt3 & 5,28 & 5,11 & 4,77 & 4,25 & 5,62 & 5,49 & 5,30 & 4,87 & 5,85 & 5,77 & 5,56 & 5,22 \\
\hline Tu3 & 5,55 & 5,35 & & 4,40 & 5,55 & 5,37 & 5,25 & 4,5 & 5,55 & 5,46 & 5,24 & 4,90 \\
\hline Tu4 & 5,35 & & & 4,24 & 5,66 & 5,57 & 5,46 & 4,65 & 5,85 & 5,81 & 5,61 & 4,96 \\
\hline Ts2 & 4,81 & 4,69 & 4,21 & 3,84 & 6,16 & 6,01 & 5,91 & 4,96 & 7,27 & 7,12 & 6,52 & 5,79 \\
\hline $\mathrm{Tl}$ & 5,28 & & & 4,14 & 5,5 & 5,40 & 5,17 & 4,37 & 5,85 & 5,69 & 5,31 & 4,64 \\
\hline Tu2 & 5,55 & 5,34 & 4,79 & 4,16 & 5,55 & 5,36 & 5,19 & 4,45 & 5,55 & 5,38 & 5,02 & 4,52 \\
\hline $\mathrm{Tt}$ & 5,55 & 5,29 & 4,52 & 3,83 & 5,55 & 5,32 & 4,95 & 3,82 & 5,55 & 5,36 & 4,63 & 3,60 \\
\hline
\end{tabular}


Tabelle 3c. Einfluss der Bodenarten (BA bei minimalem Tongehalt) auf die Temperaturleitfähigkeit $\mathrm{k}$ in Abhängigkeit vom Matrixpotenzial (pF-Wert) für die 3 Klassen der Trockenrohdichte $<1,4 \mathrm{~g} \mathrm{~cm}^{-3}, 1,4-1,6 \mathrm{~g} \mathrm{~cm}^{-3}$ und $>1,6 \mathrm{~g} \mathrm{~cm}^{-3}$

Table 3c. Impact of soil texture classes (BA with minimum clay content) on the thermal diffusivity $\mathrm{k}$ depending on matric potential ( $\mathrm{pF}$ value) for the three bulk density classes $<1.4 \mathrm{~g} \mathrm{~cm}^{-3}, 1.4-1.6 \mathrm{~g} \mathrm{~cm}^{-3}$ und $>1.6 \mathrm{~g} \mathrm{~cm}^{-3}$

\begin{tabular}{|c|c|c|c|c|c|c|c|c|c|c|c|c|}
\hline & \multicolumn{4}{|c|}{$\begin{array}{c}\mathrm{k}\left[10^{-7} \mathrm{~m}^{2} \mathrm{~s}^{-1}\right] \\
\text { bei } \rho_{\mathrm{t}}<1,4 \mathrm{~g} \mathrm{~cm}^{-3}\end{array}$} & \multicolumn{4}{|c|}{$\begin{array}{c}\mathrm{k}\left[10^{-7} \mathrm{~m}^{2} \mathrm{~s}^{-1}\right] \\
\text { bei } \rho_{\mathrm{t}}=1,4-1,6 \mathrm{~g} \mathrm{~cm}^{-3}\end{array}$} & \multicolumn{4}{|c|}{$\begin{array}{c}\mathrm{k}\left[10^{-7} \mathrm{~m}^{2} \mathrm{~s}^{-1}\right] \\
\text { bei } \rho_{\mathrm{t}}>1,6 \mathrm{~g} \mathrm{~cm}^{-3}\end{array}$} \\
\hline & $\mathrm{pF}$ & $\mathrm{pF}$ & $\mathrm{pF}$ & $\mathrm{pF}$ & & $\mathrm{pF}$ & $\mathrm{pF}$ & & & & $\mathbf{p F}$ & $\mathrm{pF}$ \\
\hline BA & $-\infty$ & 1,8 & & & & & & & & & & \\
\hline Ss & 5,5 & 75 & & 8,0 & ,94 & 9,78 & 9,95 & 10 , & 8,45 & & & \\
\hline Su2 & & & & & & & & & & & & \\
\hline S12 & & & & & & & & & & & & \\
\hline $\mathrm{Sl} 3$ & & & & & & & & & & & & \\
\hline St2 & & & & & & & & & & & & \\
\hline & & & & & & & & & & & & \\
\hline & & & & & & & & & & & & \\
\hline & & & & & & & & & & & & \\
\hline 31 & & & & & & & & & & & & \\
\hline & & & & & & & & & & & & \\
\hline & & & & & & & & & & & & \\
\hline & & & & & & & & & & & & \\
\hline & & & & & & & & & & & & \\
\hline $\mathrm{Lt}$ & & & & & & & & & & & & \\
\hline Lts & & & & & & & & & & & & \\
\hline & & & & & & & & & & & & \\
\hline Ts3 & & & & & & & & & & & & \\
\hline $\mathrm{Uu}$ & & & & & & & & & & & & \\
\hline Us & & & & & & & & & & & & \\
\hline & & & & & & & & & & & & \\
\hline & & & & & & & & & & & & \\
\hline Ul & & & & & & & & & & 73 & 7,61 & \\
\hline & & & & & & & & & & & & \\
\hline $\mathrm{Lu}$ & & & & & 6,94 & & 7,66 & & & 9,441 & & \\
\hline & & & & & & & & & & 9,18 & 9,35 & \\
\hline & & & & & & & & & & 6,59 & 6,51 & \\
\hline Tu4 & & & & & & & & & & 22 & 6,17 & \\
\hline Ts2 & & & & & & & & & & 6,66 & 6,61 & \\
\hline $\mathrm{Tl}$ & & & & & & & & & & & & \\
\hline & & & & & & & & & & & & \\
\hline $\mathrm{Tt}$ & 5,55 & & 4,91 & 4,46 & 5,55 & 5,41 & 5,19 & 4,45 & 5,55 & 5,44 & 4,99 & 4,2 \\
\hline
\end{tabular}

tonreicher die Bodenart generell ist. Da aber gleichzeitig die typischen Kurvenverläufe mittlerer Tongehalte (Abb. 4) bei den maximalen innerhalb der Bodenart möglichen Tongehalten auch noch zu höheren $\mathrm{pF}-$ Werten verschoben sind, kann vermutlich von einem für die Berechnung der Temperaturleitfähigkeit relevanten Feinporen- und damit Sättigungsgradeffekt ausgegangen werden. Dies abschließend zu beurteilen, erfordert weitere detaillierte Untersuchungen. Ungeachtet dieser offenen Fragestellungen und fehlenden Antworten hinsichtlich des tatsächlichen Einflusses der Bodenstruktur und der damit gekoppelten Toninteraktionen kann der mit dem Ansatz von Xie et al. (2018) erstellte Datensatz auf der Makroskala für die typischen Bodenarten zur Vorhersage der thermischen Kenngrößen C, $\lambda$ und $\mathrm{k}$ mit ausreichender Genauigkeit flächendeckend herangezogen werden. Damit können folglich bereits vor Beginn der eigentlichen Baumaßnahmen die Standortverhältnisse hinsichtlich der Wärmehaushaltskenngrößen eingeordnet werden, indem während der Bohrstockkartierung im Feld eine Ansprache der Lagerungsdichte (zur Ableitung der Trockenrohdichte) und der aktuellen Wassergehalte bzw. eine Ableitung der Matrixpotenziale vorgenommen wird. Wenn außerdem die anhand der langjährigen Klimaaufzeichnungen für das Untersuchungsgebiet bekannten hydraulischen Bedingungen bekannt sind, ist es auch denkbar, dass bereits vor dem Eingriff in den Boden die Folgen und die langfristig zu planenden Maßnahmen je nach Standorteigenschaften und Ansprüchen der Landwirte quantifiziert werden. Damit könnten auch in Umweltverträglichkeitsprüfungen konkrete Maßnahmen benannt werden, die auf eine Minimierung der Bodenbelastung und mögliche Schädigung hinzielen.

Abschließend kann zusammenfassend festgestellt werden, dass die in der „KA5“ aus einer großen Anzahl von Bodenprofildaten zusammengestellten Werte der Porengrößenverteilung und der entsprechenden Körnungen (Bodenarten) als gute Grundlage für die bisher fehlende Ableitung von thermischen Kenngrößen herangezogen werden können.

\section{Schlussfolgerung}

Auf der Grundlage der in der „KA5“ zusammengestellten Werte der Porengrößenverteilung für die einzelnen Bodenarten lassen sich für unterschiedliche Trockenrohdichtebereiche mithilfe des mathematischen Modells von Xie et al. (2018) die Wärmeleitfähigkeit, volumetrische Wärmekapazität und Temperaturleitfähigkeit in Abhängigkeit vom Matrixpotenzial (pF-Wert) berechnen. Innerhalb jeder Texturklasse (Bodenart) beeinflusst die mögliche Spannbreite von Ton, Schluff und Sand in Kombination mit der Trockenrohdichte auch die Temperaturleitfähigkeit. Um den Einfluss von Bodenstruktur sowie Porenkontinuität auf thermische Kennwerte noch zu berücksichtigen, sind weitere Datensätze zu aus In-situ-Messungen abgeleiteten Kenngrößen erforderlich.

Im Hinblick auf eine mögliche Anwendung im Zusammenhang mit der Stromkabelverlegung im Boden können anhand der Bohrstockkartierung bereits vor Beginn der Baumaßnahmen Aussagen zu standortspezifischen thermisch-hydraulischen Prozessen abgeleitet und Vorgaben 
zu Ausgleichs- oder/und Schutzmaßnahmen seitens der Betreiber und auch aus der Sicht der Flächenbesitzer definiert werden.

\section{Literatur}

Ad hoc AG Boden (2005): Bodenkundliche Kartieranleitung. 5. Aufl. E. Schweizerbart, Hannover.

Amelung, W., Blume, H. P., Fleige, H., Horn, H., Kandeler, E., Kögel-Knabner, I., Kretzschmar, R., Stahr, K. und B. M. Wilke (2018): Scheffer/Schachtschabel Lehrbuch der Bodenkunde. 17. Aufl., Springer Spektrum, Heidelberg.

Campbell, G. S., Calissendorff, C. und J. H. Williams (1991): Probe for measuring soil specific heat using a heat-pulse method. Soil Science Society of America Journal 55, 291-293.

Chen, S., Wang, J., Zhang, T. und H. Zhenghua (2020): Climatic, soil, and vegetation controls of the temperature sensitivity (Q10) of soil respiration across terrestrial biomes. Global Ecology and Conservation 22, e00955.

de Vries, D. A. (1963): Thermal properties of soils. In: Van Wijk, W. R. (Hrsg.): Physics of plant environment. North-Holland Publ. Co., Amsterdam.

Dehner, U. und N. Feldwisch (2015): Boden und Energiewende - Trassenbau, Erdverkabelung und Erdwärme. Springer, Wiesbaden.

Ehlers, W. (1996): Wasser in Boden und Pflanze - Dynamik des Wasserhaushalts als Grundlage von Pflanzenwachstum und Ertrag. Ulmer, Stuttgart.

Gebhardt, S., Zink, A., Fleige, H. und R. Horn (2012): Bodenschutz auf Linienbaustellen am Beispiel der Erdverkabelung für den landseitigen Netzanschluss. Bodenschutz 01/12.

Hartge, K. H. und R. Horn (2016): Essential Soil Physics. Schweizerbart., Stuttgart.

Heusinkveld, B. G., Jacobs, A., Holtslag, A. und S. M. Berkowicz (2004): Surface energy balance closure in an arid region: Role of soil heat flux. Agricultural and Forest Meteorology 122, 21-37.

Horn, R. und A. Smucker (2005): Structure formation and its consequences for gas and water transport in unsaturated arable and forest soils. Soil and Tillage Research $82,5-14$.
Horn, R., Mordhorst, A. und H. Fleige (2021): Consequences of gas pipeline hauling on changes in soil properties over 3 years. Soil and Tillage Research 211, 105002.

Horton, R. (2002): Soil thermal diffusivity. In: Dane, J. H. und G. C. Topp (Hrsg.): Methods of Soil Analysis, Part 4 - Physical Methods. Soil Science Society of America Book Series, Vol. 5, Soil Society of America, 349-360.

Jackson, R. D. und S. A. Taylor (1986): Thermal conductivity and diffusivity. Methods of Soil Analysis: Part 1 Physical and Mineralogical Methods. Soil Science Society of America, 945-956.

Kaune, A., Türk, T. und R. Horn (1993): Alteration of soil thermal properties by structure formation. Journal of Soil Science 44, 231-249.

Kirkham, M. B. (2004): Principles of Soil and Plant Water Relations. Elsevier Academic Press, Amsterdam.

Lu, S., Ren, T. S., Gong, Y. S. und R. Horton (2007): An improved model for predicting soil thermal conductivity from water content. Soil Science Society of America Journal 71, 8-14.

Markert, Peters, A. und G. Wessolek (2016): Analysis of the evaporation method to obtain soil thermal conductivity data in the full moisture range. Soil Science Society of America Journal 80, 275-283.

Trinks, S. (2010): Einfluss des Wasser- und Wärmehaushaltes von Böden auf den Betrieb erdverlegter Energiekabel. Dissertation, Technische Universität Berlin.

Trinks, S., Kluge, B., Wessolek, G. und M. Köhler (2013): CableEARTH: Ein neues Berechnungsverfahren zur Optimierung der Strombelastbarkeit erdverlegter Energiekabel. Netzpraxis 52 (12), 51-58.

Wessolek, G., Trinks, S., Kluge, B., Bohne, K. und N. Markwardt (2017): Bewertung der Bodenerwärmung durch Erdkabeltrassen. In: Wissenschaftsdialog, Bundesnetzagentur, 82-92.

Winkler, J. P, Cherry, R. S. und W. H. Schlesinger (1996): The Q10 relationship of microbial respiration in a temperate forest soil. Soil Biology and Biochemistry 28, $1067-1072$.

Xie, X., Lu, L., Ren, T. und R. Horton (2018): Empirical Model for Estimating Soil Thermal Diffusivity from Texture, Bulk Density, and Degree of Saturation. Journal of Hydrometeorology 19, 445-457.

Zink, A., Fleige, H., Gebhardt, S. und R. Horn (2013): Verification of Subsoil Compaction on Cable Construction Sites. Advances in Geoecology 42, 148-163. 